\title{
Combined Sacral Nerve Roots Stimulation and Low Thoracic Spinal Cord Stimulation for the Treatment of Chronic Pelvic Pain
}

\author{
Samer Abdel-Aziz, Ahmed Ghaleb \\ Department of Anesthesiology and Pain Medicine, University of Arkansas for Medical Sciences, Little Rock, USA \\ Email: sabdelaziz@uams.edu
}

Received 28 January 2014; revised 28 February 2014; accepted 6 April 2014

Copyright (C) 2014 by authors and Scientific Research Publishing Inc.

This work is licensed under the Creative Commons Attribution International License (CC BY). http://creativecommons.org/licenses/by/4.0/

c) (7) Open Access

\begin{abstract}
Some pelvic pain syndromes are very resistant to medical treatment. Several studies have demonstrated that sacral neuromodulation, which has been successfully used for the treatment of bladder dysfunction, incontinence, urinary retention and urinary frequency [1]-[3], can be successfully used for the treatment of chronic pelvic pain [4]-[7]. Several studies have also demonstrated significant involvement of dorsal column pathways in the transmission of visceral pelvic pain [8] and the successful use of spinal cord stimulation for the treatment of chronic pelvic pain [9]. We report three cases of severe chronic pelvic pain that failed conservative treatment modalities. Placement of a combined sacral nerve roots stimulator and a low thoracic spinal cord stimulator resulted in a significant pain relief and improvement in daily life activities. We believe that this combination may help patients suffering from chronic pelvic pain resistant to medical management.
\end{abstract}

\section{Keywords}

Chronic Pelvic Pain, Sacral Nerve Roots Stimulation, Thoracic Spinal Cord Stimulation

\section{Introduction}

Some pelvic pain syndromes are very resistant to medical treatment and seem to have neuropathic pain features. Several studies have demonstrated that sacral neuromodulation, which has been successfully used for the treatment of bladder dysfunction, incontinence, urinary retention and urinary frequency [1]-[3], can be successfully used for the treatment of chronic pelvic pain [4]-[7]. Several studies have also demonstrated significant involvement of dorsal column pathways in the transmission of visceral pelvic pain [8] and the successful use of spinal cord stimulation for the treatment of chronic pelvic pain [9]. In this retrospective case series we describe the use 
of a combination of sacral nerve roots stimulation and thoracic spinal cord stimulation in the treatment of severe chronic pelvic pain.

\section{Case Series}

We report three cases in which the use of a combined sacral nerve roots stimulator and a low thoracic spinal cord stimulator provided successful treatment for patients with chronic pelvic pain.

The first patient was a 33-year-old female with endometriosis causing severe pelvic pain, dyspareunia and pain with bowel movements for several years. She underwent eight pelvic surgeries including a hysterectomy which did not give her any pain relief. Several pain medication regimens failed to relief her pain or improve her quality of life. A differential epidural steroid injection failed to give her any pain relief. A hypogastric plexus block gave her a significant but non-sustained relief. After passing a psychological evaluation, decision was made to proceed with a combined sacral nerve roots stimulator and thoracic spinal cord stimulator trail.

The second patient was a 31 year-old male with severe pelvic and scrotal pain for many years, multiple pain medication regimens failed to relief his pain. An iliohypogastric and femoral nerve blocks gave him a significant but non-sustained pain relief. After passing a psychological evaluation, decision was made to proceed with a combined sacral nerve roots stimulator and thoracic spinal cord stimulator trail.

The third patient was a 53 year-old female with severe lower pelvic and perineal pain for many years. Several regimens of pain medications failed to give her pain relief. A ganglion Impar block gave her a good but temporary pain relief. After passing a psychological evaluation, decision was made to proceed with a combined sacral nerve roots stimulator and thoracic spinal cord stimulator trail.

In all three patients, a 14 gauge Tuohy needle was introduced using a right paramedian approach, advanced into the epidural space at L1-L2 level at an angle of 30 - 45 degrees to the skin. The epidural space was confirmed using a loss of resistance to air technique and by passing a guide wire. The spinal cord stimulator lead was advanced caphaladly with maneuvering, positioned over the dorsal column of the spinal cord. The most cephalad electrode was placed at the superior endplate of the T9 vertebral body (Picture 1). A second 14 gauge Tuohy needle was introduced using a left paramedian approach, advanced into the epidural space at L1-L2 level at an angle of 30 - 45 degrees to the skin. The epidural space was confirmed using a loss of resistance to air technique and by passing a guide wire. A second lead was advanced caudally with maneuvering, positioned over the sacral canal to lie next to S1, 2 and 3 nerve roots (Picture 2). The leads were then connected to the programming extension and passed to the company representative. It was programmed to give the patients maximal pain coverage.

All patients reported immediate pain relief. On follow up, five days later, they all reported more than 50\% pain relief and improvement in activities of daily living. They all proceeded with permanent stimulators implantation (Picture 3).

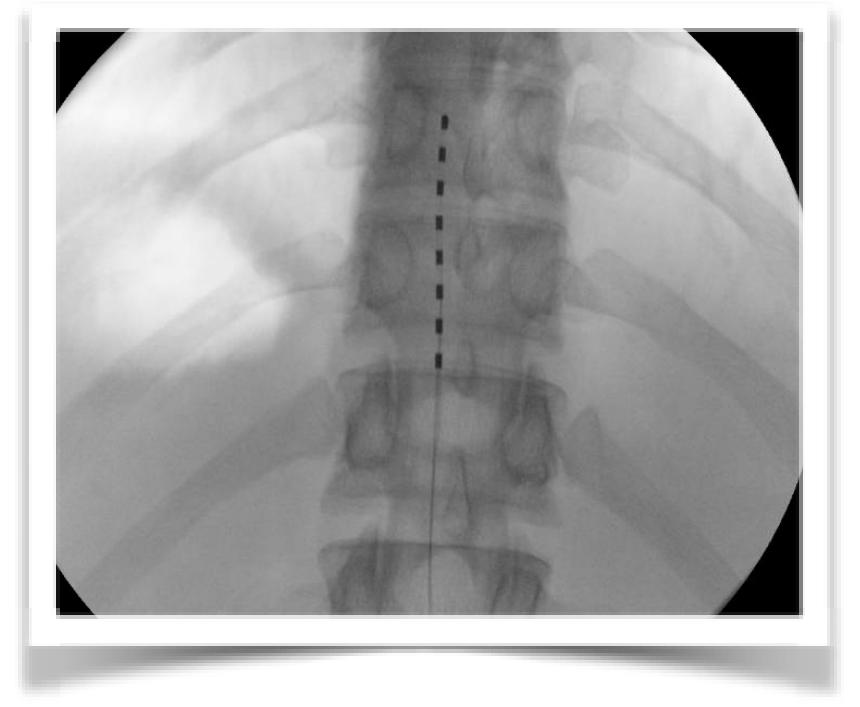

Picture 1. Low thoracic SCS lead. 


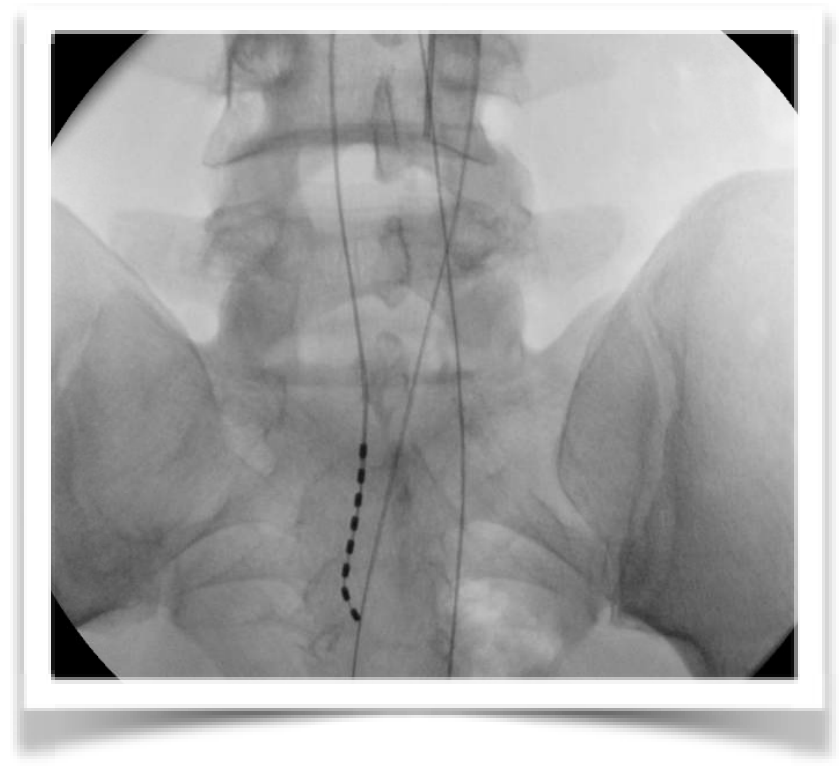

Picture 2. Sacral nerve roots stimulator lead.

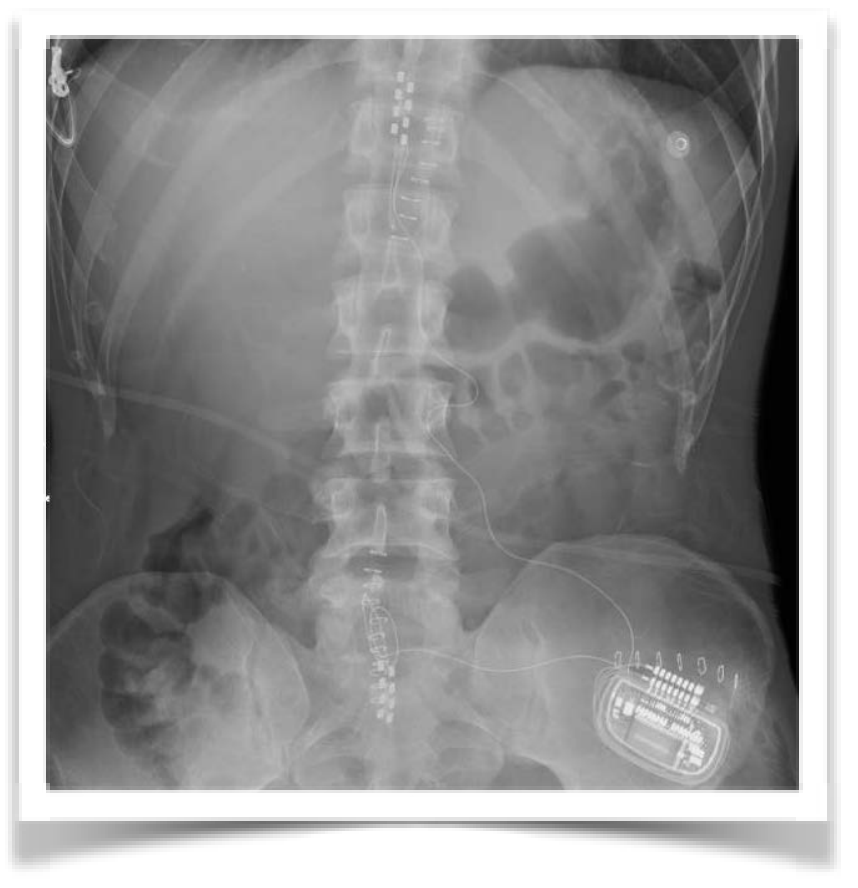

Picture 3. Permanent thoracic SCS and SNS.

\section{Discussion}

The pelvis has visceral and somatic structures innervated by the sympathetic (T12-L2), parasympathetic (S2-S4) and somatic (S2-S4) nervous systems. This complex innervation makes diagnosis of pain in this region very difficult which may delay the diagnosis and coverts an acute pain condition to a chronic pelvic pain. Chronic pelvic pain (CPP) affects one in seven women in the reproductive age in the United States [10]. While some patients with CPP respond to medical treatment many require interventional procedures (hypogastric plexus block, ganglion Impar block, pudendal nerve block) to relief their pain, and many undergo surgical procedures hoping for some pain relief. In addition to the complex innervation of that region, the fact that the exact etiology of CPP 
is not known makes treatment more difficult, it could be centralized, neuropathic or sympathetically driven, some even proposed the idea that CPP is a form of chronic regional pain syndrome (CRPS) [11].

Sacral neuromoduation has been successfully used in treating many urological conditions. In 1997, the FDA approved the use of epidural sacral nerve root stimulation for the treatment of urinary urgency, frequency, urge incontinence, and retention [1]-[3]. Sacral nerve root stimulation has also been used in treating pelvic pain, many studies were able to demonstrate reductions in both pain and narcotic requirements with the use of sacral nerve root stimulation [4]-[7]. It has proven to be an effective treatment of many pelvic pain syndromes including Interstitial cystitis, painful bladder syndrome, chronic prostatitis, coccygodynia, vulvodynia, anorectal pain, and even pelvic pain from general pelvic floor dysfunction and spinal cord infarction [12]-[14]. However many studies reported that the pattern of referred pain remains refractory to that modality, and that resistance develops over time due to epidural scarring [15] [16].

Thoracic spinal cord stimulation has been used to treat CPP [9]. The exact mechanism of visceral pelvic pain suppression by spinal cord stimulation remains unclear. One possible mechanism is the modulation of the midline dorsal column pathway that mediates the perception of visceral pelvic pain [17]. Another possible mechanism is suppressing the sympathetic outflow to the pelvis [11].

Our group of patients had severe pelvic pain not responding to multiple medication regimens. Several procedures did not provide a long lasting pain relief. The combination of the sacral nerve roots stimulator and the low thoracic spinal cord stimulator provided more than 50\% pain relief and an improvement in activities of daily living, which is considered a success in these patients with resistant chronic severe pain.

\section{Conclusion}

We believe that epidural sacral nerve roots stimulation may help patients suffering from chronic pelvic pain resistant to medical management. Adding a low thoracic SCS may give a better result as it may take care of pain escaping the sacral lead, suppress the referred pattern of pain or decrease pain by suppressing the sympathetic outflow to the pelvis.

\section{References}

[1] Van Kerrebroeck, P.E. (2010) Advances in the Role of Sacral Nerve Neuromodulation in Lower Urinary Tract Symptoms. International Urogynecology Journal, 21, S467-S474.

[2] Mayer, R.D. and Howard, F.M. (2008) Sacral Nerve Stimulation: Neuromodulation for Voiding Dysfunction and Pain. Neurotherapeutics, 5, 107-113. http://dx.doi.org/10.1016/j.nurt.2007.10.063

[3] Powell, C.R. and Kredert, K.J. (2010) Long-Term Outcomes of Urgency-Frequency Syndrome Due to Painful Bladder Syndrome Treated with Sacral Neuromodulation and Analysis of Failures. Journal of Urology, 183, 173-176. http://dx.doi.org/10.1016/j.juro.2009.08.142

[4] Maher, C.F., Carey, M.P., Dwyer, P.L., et al. (2001) Percutaneous Sacral Nerve Root Neuromodulation for Intractable Interstitial Cystitis. Journal of Urology, 165, 884-886. http://dx.doi.org/10.1016/S0022-5347(05)66551-2

[5] Seigel, S., Paszkiewicz, E., Kirkpatrick, C., et al. (2001) Sacral Nerve Stimulation in Patients with Chronic Intractable Pelvic Pain. Journal of Urology, 166, 1742-1745. http://dx.doi.org/10.1016/S0022-5347(05)65665-0

[6] Everaert, K., Devulder, J., De Muynck, M., et al. (2001) The Pain Cycle: Implications for the Diagnosis and Treatment of Pelvic Pain Syndromes. International Urogynecology Journal, 12, 9-14. http://dx.doi.org/10.1007/s001920170087

[7] Yang, K.S., Kim, Y.H., Park, H.J., et al. (2010) Sacral Nerve Stimulation for the Treatment of Chronic Intractable Anorectal Pain: A Case Report. Korean Journal of Pain, 23, 60-64. http://dx.doi.org/10.3344/kjp.2010.23.1.60

[8] Palacek, J. and Wilis, W.D. (2003) The Dorsal Column Pathway Facilitates Visceromotor Responses to Colorectal Distention after Colon Inflammation in Rats. Pain, 104, 501-507. http://dx.doi.org/10.1016/S0304-3959(03)00075-7

[9] Kapural, L., Narouze, S.N., Janicki, T.I., et al. (2006) Spinal Cord Stimulation Is an Effective Treatment for the Chronic Intractable Visceral Pelvic Pain. Pain Medicine, 7, 440-443. http://dx.doi.org/10.1111/j.1526-4637.2006.00165.x

[10] Steege, J.F. (1999) Scope of the Problem. In: Steege, J.F., Metzger, D.M., Levy, B.S., Eds., Chronic Pelvic Pain: An Integrated Approach, W.B. Saunders, Philadelphia, 9.

[11] Janicki, T.I. (2003) Chronic Pelvic Pain as a Form of Complex Regional Pain Syndrome. Clinical Obstetrics and Gynecology, 46, 797-803. http://dx.doi.org/10.1097/00003081-200312000-00009

[12] Kothari, S. (2007) Neuromodulation Approaches to Chronic Pelvic Pain and Coccydynia. Acta Neurochir Supplement, 
97, 365-371.

[13] Fariello, J.Y. and Whitmore, K. (2010) Sacral Neuromodulation for IC/PBS, Chronic Pelvic Pain, and Sexual Dysfunction. International Urogynecology Journal, 21, 1553-1558.

[14] Feler, C.A., Whitworth, L.A. and Fernandez, J. (2003) Sacral Modulation for Chronic Pain Conditions. Anesthesiology Clinics of North America, 21, 785-795. http://dx.doi.org/10.1016/S0889-8537(03)00085-3

[15] Zabihi, N., Mourtzinos, A., Maher, M.G., et al. (2008) Short-Term Results of Bilateral S2-S4 Sacral Neuromodulation for the Treatment of Refractory Interstitial Cystitis, Painful Bladder Syndrome, and Pelvic Pain. International Urogynecology Journal and Pelvic Floor Dysfunction, 19, 553-557. http://dx.doi.org/10.1007/s00192-007-0466-X

[16] Brookoff, D. and Bennett, D.S. Neuromodulation in Intractable Interstitial Cystitis and Related Pelvic Pain Syndromes. Pain Medicine, 7, S166-S184. http://dx.doi.org/10.1111/j.1526-4637.2006.00132.x

[17] Nauta, H.J., Soukup, V.M., Fabian, R.H., et al. (2000) Punctate Midline Myelotomy for the Relief of Visceral Cancer Pain. Journal of Neurosurgical Spine, 92, 125-130. http://dx.doi.org/10.3171/spi.2000.92.2.0125 